\title{
Contact poling of RKTP with silicon pillars
}

\author{
Hoda Kianirad, Andrius Zukauskas, Thomas Frisk, Carlota Canalias and Fredrik Laurell \\ Applied Physics Department, Royal Institute of Technology, Roslagstullsbacken 21, SE-106 91 Stockholm, Sweden
}

Quasi-phase-matching (QPM) is a method to get tailored efficient second order nonlinear interactions [1]. Several techniques exist for fabrication of periodic domain structures in ferroelectric crystals for QPM frequency conversion. By far, electric field poling using lithographically patterned electrodes on the z-face of the crystal is the most common one [2]. High-quality periodically inverted ferroelectric domain structures in flux grown $\mathrm{KTiOPO}_{4}(\mathrm{KTP})$ crystals were fabricated already in the late 90's using this technique [3], and recently periodic domain sizes of few hundred nanometers were fabricated in $1 \mathrm{~mm}$ thick samples thanks to the quasi-one dimensional structure of KTP. It has recently also been shown that a slight Rb doping of the KTP crystal (RKTP) facilitates the periodic poling [4]. However, fabrication of two-dimensional (2D) domain structures in RKTP has not yet been investigated. A disadvantage with the lithographic patterning is that each sample needs to be patterned individually, which is tedious and time consuming. Moreover, when it comes to the small domain features, which are required by the next generation of nonlinear optical devices, a more versatile poling technique has to be developed due to the limitations of conventional photolithography. Structured silicon has been investigated as an alternative electrode for formation of $1 \mathrm{D}$ domains by contact poling in $\mathrm{LiNbO}_{3}$ [5]. However, these electrodes were fabricated by wet etching and the sample thickness was limited to $\sim 200 \mu \mathrm{m}$.

In this work we present a new technique for 2D domain inversion in $1 \mathrm{~mm}$ thick RKTP and demonstrate the densest 2D lattice in a KTP isomorph. First, 2D periodic arrays of silicon pillars were constructed using isotropic dry etching (Fig.1 (a)). The silicon substrate was p-type with <100> orientation and a resistivity of $0.005-0.02$ $\Omega \mathrm{cm}$. Second, the silicon arrays of pillars were used as contact electrode to periodically pole the RKTP crystal (Fig.1 (b)). A regular and homogeneous domain pattern with a $5 \times 5 \mu \mathrm{m}^{2}$ period was obtained, as can be seen in Fig.1 (c). A high normalized conversion efficiency of $1.27 \% \mathrm{~W}^{-1} \mathrm{~cm}^{-1}$ was obtained for frequency doubling of a CW Ti-Sapphire laser at $894 \mathrm{~nm}$. The measured temperature bandwidth was $4.25^{\circ} \mathrm{C}$, from which we estimated an effective crystal length of $5.4 \mathrm{~mm}$, which is very close to the physical structure length of $6 \mathrm{~mm}$.

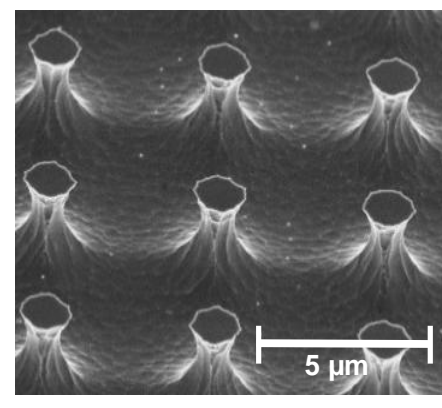

(a)

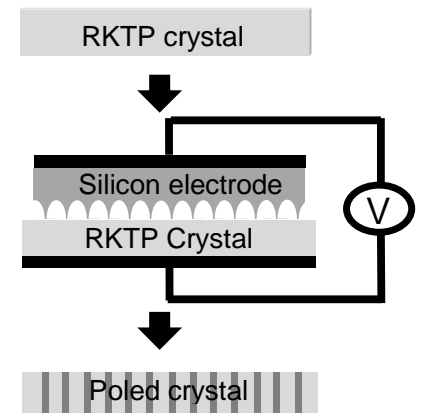

(b)

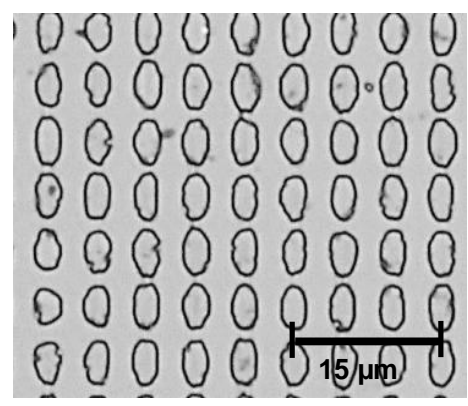

(c)

Fig. 1 (a) Silicon array of pillars fabricated by dry isotropic etching; (b) electric field poling process by using the silicon array of pillars; (c) fabricated domain structure with $5 \times 5 \mu \mathrm{m}^{2}$ period by using the array of silicon pillars as a contact electrode.

This novel poling technique has several advantages. First, the silicon electrode is reusable and there is no need for patterning each sample individually. Second, the crystalline structure of silicon provides high accuracy and reproducibility in the electrode fabrication. Finally, Si-pillar array electrodes can be designed for any desirable period or electrode geometry. Therefore, we believe that contact electrode poling can be a more convenient, flexible and suitable method for making small feature domain patterns, and it also can reduce the fabrication costs.

\section{References}

[1] J. A. Armstrong, N. Bloembergen, J. Ducuing, and P. S. Pershan, "Interactions between Light Waves in a Nonlinear Dielectric," Phys.Rev. 127, 1918 (1962).

[2] M. Yamada, N. Nada, M. Saitoh, and K. Watanabe, "First-order quasi-phase matched $\mathrm{LiNbO}_{3}$ waveguide periodically poled by applying an external field for efficient blue second-harmonic generation,” Appl. Phys. Lett. 62, 435 (1992).

[3] H. Karlsson and F. Laurell, "Electric field poling of flux grown KTiOPO 4 ," Appl. Phys. Lett. 71, 3474 (1997)

[4] A. Zukauskas, G. Strömqvist, V. Pasiskevicius, F. Laurell, M. Fokine, and C. Canalias, "Fabrication of submicrometer quasi-phasematched devices in KTP and RKTP," Opt. Mater. Express 1, 1319 (2011).

[5] H. Kintaka, K, Fujimura, M, Suhara, T and Nishihara, "Fabrication of ferroelectric-domain-inverted grating in $\mathrm{LiNbO}_{3}$ by applying voltage using etched-Si stamper electrode," Electron. Lett. 34, 880 (1998). 